\title{
Higher serum carotenoid concentrations associated with a lower prevalence of the metabolic syndrome in middle-aged and elderly Chinese adults
}

\author{
Jun Liu ${ }^{1,2}$, Wen-qi Shi ${ }^{1}$, Yi Cao ${ }^{1}$, Li-ping $\mathrm{He}^{1,3}$, Ke Guan ${ }^{1}$, Wen-hua Ling ${ }^{1}$ and Yu-ming $\mathrm{Chen}^{1 *}$ \\ ${ }^{1}$ Guangdong Provincial Key Laboratory of Food, Nutrition, and Health, Department of Medical Statistics and Epidemiology, \\ School of Public Health, Sun Yat-sen University, Guangzhou, Guangdong 510080, People's Republic of China \\ ${ }^{2}$ School of Public Health, Zunyi Medical University, Zunyi, Guizhou 563003, People's Republic of China \\ ${ }^{3}$ Guangzhou Panyu Central Hospital, Guangzhou, Guangdong 511430, People's Republic of China
}

(Submitted 19 March 2014 - Final revision received 8 July 2014 - Accepted 5 September 2014 - First published online 27 October 2014)

\section{Abstract}

The association between serum carotenoids and the metabolic syndrome (MetS) remains uncertain, and little is known about this relationship in the Chinese population. The present study examined the association between serum carotenoid concentrations and the MetS in Chinese adults. We conducted a community-based cross-sectional study in which 2148 subjects (1547 women and 601 men) aged 50-75 years were recruited in urban Guangzhou, China. Dietary data and other covariates were collected during face-to-face interviews. Blood pressure, waist circumference, blood lipids, glucose and serum carotenoids ( $\alpha$-, $\beta$-carotene, $\beta$-cryptoxanthin, lycopene and lutein/ zeaxanthin) were examined. We found dose-response inverse relationships between individual serum carotenoid concentrations and total carotenoids and the prevalence of the MetS after adjusting for potential confounders $(P$ for trend $<0 \cdot 001)$. The OR of the MetS for the highest $(v$. lowest) quartile were $0.31(95 \%$ CI $0 \cdot 20,0 \cdot 47)$ for $\alpha$-carotene, $0 \cdot 23$ (95\% CI $0 \cdot 15,0 \cdot 36)$ for $\beta$-carotene, $0 \cdot 44$ (95\% CI $0 \cdot 29$, $0 \cdot 67$ ) for $\beta$-cryptoxanthin, 0.39 (95\% CI $0 \cdot 26,0.58$ ) for lycopene, $0 \cdot 28$ (95\% CI $0 \cdot 18,0 \cdot 44$ ) for lutein + zeaxanthin and $0 \cdot 19$ (95\% CI $0 \cdot 12,0 \cdot 30)$ for total carotenoids. Higher concentrations of each individual carotenoid and total carotenoids were significantly associated with a decrease in the number of abnormal MetS components ( $P$ for trend $<0 \cdot 001-0 \cdot 023$ ). Higher serum carotenoid levels were associated with a lower prevalence of the MetS and fewer abnormal MetS components in middle-aged and elderly Chinese adults.

Key words: Carotenoids: Serum: Metabolic syndrome: Cross-sectional studies

The metabolic syndrome (MetS) is a cluster of independent risk factors that increase the risk of CVD and type 2 diabetes $^{(1,2)}$. Existing data indicate that the incidence of the MetS is rising at an alarming rate globally ${ }^{(3)}$. In China, the MetS has become an increasing public health problem due to the changes in lifestyle brought about by economic development ${ }^{(4,5)}$. Although the exact mechanism of the MetS is still being elucidated, a growing body of evidence suggests that oxidative stress plays a considerable role in its pathogenesis $^{(6-8)}$. Carotenoids are well known for antioxidant activities including quenching free radicals, reducing damage from reactive oxygen species and inhibiting lipid peroxidation $^{(9-11)}$. The Coronary Artery Risk Development in Young Adults study suggested that carotenoids could prevent obesity by decreasing oxidative stress generated by adipose tissue $^{(12)}$. Furthermore, carotenoids might inhibit proinflammatory cytokine and chemokine production, and then decrease the risk of obesity-associated pathologies, such as insulin resistance ${ }^{(13,14)}$. Thus, it was assumed that carotenoids might protect against the MetS.
An inverse association of serum carotenoids with the prevalence of the MetS has been shown in three cross-sectional studies conducted in the USA ${ }^{(15-17)}$. However, in a crosssectional study with 1073 Japanese adults, Sugiura et al. ${ }^{(18)}$ found the inverse relationship only in current smokers. Such a favourable association was not found in another cross-sectional study conducted among current male Japanese smokers ${ }^{(19)}$ and in an Australian study ${ }^{(20)}$. A randomised controlled trial (RCT) has suggested that dietary supplementation with $\beta$-carotene had no significant effect on the incidence of the MetS ${ }^{(21)}$. The discrepancies in the results found among these studies might be attributable to the differences in study populations and designs, exposure makers and covariate adjustments. Moreover, significant ethnic differences in the associations between serum carotenoids and obesity have been reported, in which much weaker associations have been observed in black than in white participants ${ }^{(12)}$. The prevalence of the MetS was much lower in the Chinese (13.7\%) than in the Caucasian (>25\%) population $^{(22,23)}$. Apart from genetic factors ${ }^{(24)}$, many environmental factors, such as diet, and other lifestyle factors might

Abbreviations: BP, blood pressure; HDL-C, HDL-cholesterol; MetS, metabolic syndrome; RCT, randomised controlled trial.

*Corresponding author: Professor Y.-m. Chen, fax +86 20 87330446, email chenyum@mail.sysu.edu.cn 
contribute to these differences ${ }^{(25,26)}$. Therefore, ethnic differences in the associations between carotenoids and the MetS could not be excluded, and the results obtained in other populations might not be well generalised to the Chinese population.

In the present cross-sectional study, we investigated the relationships between the major serum carotenoids ( $\alpha$-, $\beta$-carotene, $\beta$-cryptoxanthin, lycopene and lutein/zeaxanthin) and the prevalence of the MetS in middle-aged and elderly Chinese adults.

\section{Methods}

The present community-based cross-sectional study enrolled 3169 subjects aged 50-75 years in Guangzhou, Guangdong Province, China, from October 2008 to June 2010. Participants were required to have lived in Guangzhou for at least 5 years, and were recruited from communities in Guangzhou through advertisements, health talks and referrals. We excluded the participants who reported having confirmed cancer, CHD, stroke, Alzheimer's disease or dementia before the start of the study, or who had used medication (e.g. statin, antihypertension or anti-diabetic drugs) known to affect plasma lipids in the previous 3 months ( $n$ 201). We further excluded the participants who had missing or incomplete dietary data, had implausibly high $(\geq 16736 \mathrm{~kJ} ; \geq 4000 \mathrm{kcal})$ or low $(\leq 1674 \mathrm{~kJ} ; \leq 400 \mathrm{kcal})$ total daily energy intakes ( $n$ 14) and had missing carotenoid data ( $n$ 806). Finally, 2148 participants were included in the analysis. The present study was conducted according to the guidelines laid down in the Declaration of Helsinki and all procedures involving human subjects/patients were approved by the Ethics Committee of the School of Public Health of Sun Yat-sen University. Written informed consent was obtained from all the study participants.

\section{Data collection}

\section{Questionnaire interview}

A face-to-face interview was conducted by trained interviewers using a structured questionnaire. We collected information on the participants' sociodemographic characteristics, medication, lifestyle factors, history of disease, menopausal status (for women only), dietary intake and physical activity. Daily physical activity was estimated using a physical activity questionnaire containing nineteen items, and the metabolic equivalent intensity (excluding sleeping and sitting) was calculated ${ }^{(27)}$. Usual dietary intake was assessed by using a validated quantitative FFQ including seventy-nine items. The correlation coefficient for $\beta$-carotene was 0.55 for the short-term reproducibility, and that between the second FFQ and the multiple $24 \mathrm{~h}$ dietary records was $0 \cdot 32^{(28)}$. Daily energy intake and other nutrients were calculated on the basis of the Chinese Food Composition Table $2004^{(29)}$.

\section{Measurements of anthropometric indices and blood pressure}

Body weight and height were measured with participants wearing no shoes and light clothing. BMI was calculated as weight $(\mathrm{kg}) /$ height $^{2}\left(\mathrm{~m}^{2}\right)$. Waist circumference was measured at the mid-line between the costal margin and the iliac crest, while hip circumference was measured at the point of the maximum girth around the buttocks. Measurements of waist and hip circumferences were made twice and the mean of the two measurements was used for further analysis. Overall, two consecutive blood pressure (BP) measurements were taken from the right arm after the participants were seated for at least $10 \mathrm{~min}$. BP (systolic and diastolic) was recorded to the nearest $2 \mathrm{mmHg}$. A third measurement was taken when the discrepancy between the two measured values was larger than $4 \mathrm{mmHg}$. The mean measurements were used for subsequent analyses.

\section{Laboratory assay}

Blood samples were obtained in the morning after an overnight fast. Serum was separated after centrifugation at $1500 \mathrm{~g}$ for $15 \mathrm{~min}$ at $4^{\circ} \mathrm{C}$ within $2 \mathrm{~h}$ and stored at $-80^{\circ} \mathrm{C}$ until routine chemical analysis by standard methods. Serum total cholesterol, TAG, LDL-cholesterol, HDL-cholesterol (HDL-C) and fasting blood glucose concentrations were measured by colorimetric methods using commercial kits (Biosino Biotechnology Company Limited) on a Hitachi 7600-010 automated analyser. The $\mathrm{CV}$ was $2 \cdot 17 \%(5.03 \mathrm{mmol}$ total cholesterol $/ \mathrm{l})$, $2.86 \% \quad(1.14 \mathrm{mmol}$ TAG/1), $3.47 \% \quad(1.70 \mathrm{mmol}$ HDL-C/1), $4.67 \%$ (2.65 mmol LDL-cholesterol/1) and $2.52 \%(4.45 \mathrm{mmol} / 1$ fasting blood glucose)

We used reversed-phase HPLC to assay simultaneously the levels of $\alpha$-tocopherol, retinol, $\alpha$-carotene, $\beta$-carotene, $\beta$-cryptoxanthin, lycopene and lutein/zeaxanthin, according to the method described by Burri et al. ${ }^{(30)}$ with some modifications. In brief, $200 \mu$ l of serum sample were mixed with $500 \mu \mathrm{l}$ of ethanol-butylated hydroxytoluene solution containing $\alpha$-tocopherol acetate as the internal standard, and then mixed for $1 \mathrm{~min}$ on an orbital shaker. The carotenoids were extracted twice using $2 \mathrm{ml}$ of hexane-butylated hydroxytoluene solution. The hexane layer was separated and evaporated to dry under $\mathrm{N}_{2}$ at room temperature, and the residue was dissolved in $200 \mu$ l of the mobile phase B (acetonitrilemethanol-tetrahydrofuran-ammonium acetate 55:35:5:5, v/v). Then, $50 \mu \mathrm{l}$ of samples were injected into a $\mathrm{C}_{18}$ analytical column (Shiseido) at room temperature. Peaks were detected at a wavelength of $450 \mathrm{~nm}$ for the carotenoids by a Waters 2998 diode-array detector (Waters).

As these methods do not discriminate lutein from zeaxanthin, we used the combined concentration of lutein + zeaxanthin in the analyses. A pooled plasma sample was analysed with batches of study samples to monitor the analytic precision, with a day-to-day $\mathrm{CV}$ of approximately $7 \cdot 8 \%$ for $\alpha$-carotene, $8.6 \%$ for $\beta$-carotene, $9.7 \%$ for $\beta$-cryptoxanthin, $10.6 \%$ for lycopene and $8.0 \%$ for lutein + zeaxanthin, respectively.

\section{Definition of the metabolic syndrome}

The diagnostic criteria for the Mets are based on the 2005 definition of the International Diabetes Federation ${ }^{(31)}$ : 
(1) abdominal obesity - waist circumference $\geq 90 \mathrm{~cm}$ in men or $\geq 80 \mathrm{~cm}$ in women; (2) elevated TAG $-\mathrm{TAG} \geq 1.7 \mathrm{mmol} / \mathrm{l}$; (3) low HDL-C - HDL-C $<1.03 \mathrm{mmol} / 1$ in men or $<1.30 \mathrm{mmol} / \mathrm{l}$ in women; (4) elevated $\mathrm{BP}-\mathrm{BP} \geq 130$ / $85 \mathrm{mmHg}$ or current use of anti-hypertensive medications; (5) elevated fasting glucose - fasting blood glucose $\geq 5.6 \mathrm{mmol} / 1$, or previously diagnosed type 2 diabetes, or taking oral anti-diabetic agents or insulin.

\section{Statistical analyses}

Sample size was estimated based on the prevalence (13.7\%) of the MetS in China in $2005^{(4)}$ and the reported OR (0.76) of the MetS for the highest ( $v$. lowest) quartile of serum carotenoids ${ }^{(20)}$. In each group, 537 subjects would provide $80 \%$ power to detect the association (OR 0.76) at the significance of 0.05 .
Data are presented as means and standard deviations for continuous variables and as frequencies for categorical variables. We used $\chi^{2}$ and $t$ tests, respectively, to compare the categorical and continuous variables of the participants' characteristics by MetS status (yes/no). To achieve an approximately normal distribution, logarithmic transformation was used for energy intake, while square root transformation was applied to other dietary factors. Dietary intakes were adjusted for total energy intake using the residual method. Analyses were performed on each serum carotenoid separately, and on the five carotenoids combined. Logistic regression analyses were used to compute the OR and 95\% CI of the MetS for the 2nd-4th quartiles of serum carotenoid concentrations, using the lowest quartile as the referent. The mean among the categories of the number of abnormal MetS components ( 0,1 , 2, 3 and 4) was also compared using ANCOVA. The relationships

Table 1. Characteristics of the study participants* (Mean values and standard deviations)

\begin{tabular}{|c|c|c|c|c|c|}
\hline & \multicolumn{2}{|c|}{ MetS $^{-} \dagger(1903)$} & \multicolumn{2}{|c|}{ MetS $^{+} \ddagger(245)$} & \multirow[b]{2}{*}{$P$} \\
\hline & Mean & SD & Mean & SD & \\
\hline Age (years) & 57.4 & $5 \cdot 0$ & $58 \cdot 6$ & 4.9 & $<0.001$ \\
\hline BMI $\left(\mathrm{kg} / \mathrm{m}^{2}\right)$ & $22 \cdot 9$ & $2 \cdot 9$ & $25 \cdot 9$ & $2 \cdot 7$ & $<0.001$ \\
\hline Waist circumference $(\mathrm{cm})$ & $81 \cdot 3$ & $8 \cdot 7$ & $90 \cdot 6$ & $6 \cdot 8$ & $<0.001$ \\
\hline Systolic blood pressure (mmHg) & 122 & 17 & 136 & 17 & $<0.001$ \\
\hline Diastolic blood pressure $(\mathrm{mmHg})$ & 77 & 10 & 87 & 10 & $<0.001$ \\
\hline Physical activity (MET-h/d)§ & $22 \cdot 8$ & 6.5 & $21 \cdot 0$ & $5 \cdot 2$ & 0.203 \\
\hline \multicolumn{6}{|l|}{ Serum lipids $(\mathrm{mmol} / \mathrm{l})$} \\
\hline Fasting blood glucose & 4.62 & 0.80 & $5 \cdot 24$ & 1.34 & $<0.001$ \\
\hline TAG & 1.45 & 0.92 & $2 \cdot 18$ & 1.29 & $<0.001$ \\
\hline Total cholesterol & 5.45 & 1.09 & 5.41 & 1.01 & 0.564 \\
\hline HDL-cholesterol & 1.42 & 0.33 & $1 \cdot 12$ & 0.21 & $<0.001$ \\
\hline LDL-cholesterol & 3.63 & 0.92 & 3.63 & 0.90 & 0.956 \\
\hline \multicolumn{6}{|l|}{ Serum antioxidant $(\mu \mathrm{mol} / \mathrm{l})$} \\
\hline$\alpha$-Tocopherol & $33 \cdot 3$ & $11 \cdot 1$ & $35 \cdot 6$ & $14 \cdot 0$ & 0.003 \\
\hline Retinol & 2.35 & 0.66 & 2.45 & 0.61 & 0.014 \\
\hline$\alpha$-Carotene & 0.044 & 0.033 & 0.033 & 0.022 & $<0.001$ \\
\hline$\beta$-Carotene & 0.622 & 0.455 & 0.437 & 0.313 & $<0.001$ \\
\hline$\beta$-Cryptoxanthin & 0.105 & 0.097 & 0.080 & 0.067 & $<0.001$ \\
\hline Lycopene & 0.110 & 0.076 & 0.097 & 0.076 & 0.011 \\
\hline Lutein + zeaxanthin & 0.484 & 0.240 & 0.415 & 0.231 & $<0.001$ \\
\hline \multirow[t]{2}{*}{ Total carotenoids\| } & 1.37 & 0.73 & 1.06 & 0.56 & $<0.001$ \\
\hline & $n$ & $\%$ & $n$ & $\%$ & \\
\hline Education (years) & & & & & $<0.001$ \\
\hline$<9$ & 514 & $27 \cdot 0$ & 96 & 39.2 & \\
\hline $9-12$ & 913 & $48 \cdot 0$ & 98 & $40 \cdot 0$ & \\
\hline$>12$ & 476 & $25 \cdot 0$ & 51 & $20 \cdot 8$ & \\
\hline Household income (yuan/month per person) & & & & & 0.337 \\
\hline$<2000$ & 645 & $33 \cdot 9$ & 72 & $29 \cdot 4$ & \\
\hline $2000-3000$ & 892 & $46 \cdot 9$ & 125 & $51 \cdot 2$ & \\
\hline$>3000$ & 366 & $19 \cdot 3$ & 48 & $19 \cdot 4$ & \\
\hline Smokerף & 265 & $13 \cdot 9$ & 30 & $12 \cdot 2$ & 0.493 \\
\hline Alcohol drinking ${ }^{\star *}$ & 108 & $5 \cdot 7$ & 16 & 6.5 & 0.662 \\
\hline
\end{tabular}

MetS, metabolic syndrome; MET, metabolic equivalent.

${ }^{*}$ Continuous and categorical variables were described by means and standard deviations or numbers and percentages, and evaluated by $t$ test and $\chi^{2}$ test, respectively, to compare the categorical and continuous variables of the participants with and without the MetS.

$\dagger$ Participants without the MetS.

$\ddagger$ Participants with the MetS.

$\S$ Physical activities included daily occupational, leisure-time and household chores, evaluated by MET-h/d.

$\|$ Total carotenoids indicate the sum of $\alpha$-carotene, $\beta$-carotene, $\beta$-cryptoxanthin, lycopene and lutein + zeaxanthin.

I Smokers were defined as having smoked at least one cigarette daily for at least six consecutive months.

** Alcohol drinkers were defined as having had wine (beer, white wine and red wine) at least once per week for at least six consecutive months. 
between the carotenoids and the MetS were examined after adjusting for age, sex and energy intake (model 1), and further controlling for other covariates (model 2), including marital status, education level, occupation (light, moderate and heavy physical activity), household income, smoking status, alcohol drinking, physical activity, dietary intakes of fibre, cholesterol, SFA, vitamin $\mathrm{C}, \alpha$-tocopherol and $\beta$-carotene, and serum levels of retinol and $\alpha$-tocopherol. $P$ for the linear trend was estimated by treating quartiles or category number as continuous variables. We performed subgroup analyses, respectively, stratified by age, sex, alcohol drinking, smoking status, education level and household income, and tested their interactions. Considering type I error caused by multiple comparisons, significance levels were adjusted by using Bonferroni correction ( $\alpha=0 \cdot 05 /$ number of tests). All statistical analyses were performed using SPSS for Windows (version 13.0; SPSS, Inc.).

\section{Results}

In the present community-based cross-sectional study, the prevalence of the MetS was estimated as $11.4 \%$. The demographic and health-related characteristics are presented in Table 1. As expected, participants with the MetS had elevated BMI, waist circumference, BP, TAG and fasting blood glucose and lower HDL-C concentrations than those without the MetS $(P<0 \cdot 001)$. Significant differences were also evident by age group and educational level $(P<0 \cdot 001)$. In addition, participants with the MetS had lower serum carotenoids but higher retinol and $\alpha$-tocopherol concentrations than those without the MetS ( $P$ range $<0 \cdot 001-0 \cdot 014)$. No difference was detected in the remaining characteristics (e.g. household income, smoking status and alcohol drinking) of the participants.

Consistent inverse associations of each individual level and total amount of the five carotenoids were observed with the prevalence of the MetS in the two models (Table 2). In the maximum-adjusted model, OR of the MetS for the highest ( $v$. lowest) quartile were 0.31 (95\% CI 0.20, 0.47) for $\alpha$-carotene, 0.23 (95\% CI $0.15,0.36)$ for $\beta$-carotene, 0.44 (95\% CI 0.29 , 0.67 ) for $\beta$-cryptoxanthin, 0.39 (95\% CI $0 \cdot 26,0.58)$ for lycopene, $0 \cdot 28(95 \%$ CI $0 \cdot 18,0 \cdot 44)$ for lutein + zeaxanthin and $0 \cdot 19$ (95\% CI $0 \cdot 12,0.30)$ for total carotenoids (all $P$ for trend $<0.001)$. Base on the study size analysed, we had $\geq 86 \%$ power to detect the associations $(\mathrm{OR} \leq 0.44)$ at an $\alpha$ value of $0.008(=0 \cdot 05 / \mathrm{six}$ tests $)$. The covariate-adjusted means of serum carotenoid concentrations by the number of abnormal MetS components are shown in Table 3. The serum levels of each individual carotenoid and their combination decreased significantly as the number of abnormal MetS components increased ( $P$ for trend range $<0 \cdot 001-0 \cdot 023$ ).

We examined the association between carotenoids and the MetS across different subgroups stratified by sex, smoking status and alcohol drinking (Table 4). Inverse associations between the MetS and total carotenoids and each individual carotenoid were observed only among non-smokers or nondrinkers. No significant interactions were found between carotenoids and age, sex, alcohol drinking, smoking status, education level and household income (see online supplementary Table S1), as they were related to the prevalence of the MetS ( $P$ for interaction $>0.003, \alpha=0.05 /$ eighteen tests).

Table 2. Metabolic syndrome by the quartiles of serum carotenoid concentrations

(Odds ratios and $95 \%$ confidence intervals; $n$ 537)

\begin{tabular}{|c|c|c|c|c|c|c|c|c|}
\hline & \multirow{2}{*}{$\frac{\text { Quartile } 1}{\text { OR }}$} & \multicolumn{2}{|c|}{ Quartile 2} & \multicolumn{2}{|c|}{ Quartile 3} & \multicolumn{2}{|c|}{ Quartile 4} & \multirow[b]{2}{*}{$P$ for trend } \\
\hline & & OR & $95 \% \mathrm{Cl}$ & OR & $95 \% \mathrm{Cl}$ & OR & $95 \% \mathrm{Cl}$ & \\
\hline$\alpha$-Carotene $(\mu \mathrm{mol} / \mathrm{l})$ & 0.015 & \multicolumn{2}{|r|}{0.027} & \multicolumn{2}{|c|}{0.042} & \multicolumn{2}{|c|}{0.086} & \\
\hline Case $(n)$ & 85 & \multicolumn{2}{|r|}{72} & \multicolumn{2}{|r|}{54} & \multicolumn{2}{|c|}{34} & \\
\hline Model $1^{*}$ & 1.00 & 0.80 & $0.56,1.12$ & 0.54 & $0.38,0.79$ & 0.33 & $0.21,0.50$ & $<0.001$ \\
\hline Model $2 \dagger$ & 1.00 & 0.81 & $0.58,1.15$ & 0.55 & $0.38,0.80$ & 0.31 & $0.20,0.47$ & $<0.001$ \\
\hline$\beta$-Carotene $(\mu \mathrm{mol} / \mathrm{l})$ & 0.18 & \multicolumn{2}{|r|}{0.38} & \multicolumn{2}{|r|}{0.62} & \multirow{2}{*}{\multicolumn{2}{|c|}{$\begin{array}{c}1.22 \\
35\end{array}$}} & \\
\hline Case $(n)$ & 98 & \multicolumn{2}{|r|}{63} & \multicolumn{2}{|r|}{49} & & & \\
\hline Model 1 & 1.00 & 0.55 & $0.39,0.78$ & 0.39 & $0.27,0.57$ & 0.26 & $0.17,0.40$ & $<0.001$ \\
\hline Model 2 & 1.00 & 0.52 & $0.37,0.75$ & 0.36 & $0.24,0.53$ & 0.23 & $0.15,0.36$ & $<0.001$ \\
\hline$\beta$-Cryptoxanthin $(\mu \mathrm{mol} / \mathrm{l})$ & 0.032 & \multicolumn{2}{|r|}{0.055} & \multicolumn{2}{|r|}{0.095} & \multicolumn{2}{|c|}{0.226} & \\
\hline Case $(n)$ & 81 & \multicolumn{2}{|r|}{65} & \multicolumn{2}{|r|}{58} & \multicolumn{2}{|r|}{41} & \\
\hline Model 1 & 1.00 & 0.71 & $0.50,1.01$ & 0.62 & $0.43,0.89$ & 0.41 & $0.27,0.61$ & $<0.001$ \\
\hline Model 2 & 1.00 & 0.74 & $0.52,1.08$ & 0.68 & $0.47,0.99$ & 0.44 & $0.29,0.67$ & $<0.001$ \\
\hline Lycopene $(\mu \mathrm{mol} / \mathrm{l})$ & 0.041 & \multicolumn{2}{|r|}{0.075} & \multicolumn{2}{|r|}{0.110} & \multicolumn{2}{|c|}{0.208} & \\
\hline Case $(n)$ & 89 & \multicolumn{2}{|r|}{57} & \multicolumn{2}{|r|}{51} & \multicolumn{2}{|c|}{48} & \\
\hline Model 1 & 1.00 & 0.54 & $0.38,0.78$ & 0.46 & $0.32,0.67$ & 0.43 & $0.29,0.63$ & $<0.001$ \\
\hline Model 2 & 1.00 & 0.56 & $0.39,0.80$ & 0.45 & $0.31,0.67$ & 0.39 & $0.26,0.58$ & $<0.001$ \\
\hline Lutein + zeaxanthin $(\mu \mathrm{mol} / \mathrm{l})$ & 0.22 & \multicolumn{2}{|r|}{0.37} & \multicolumn{2}{|r|}{0.51} & \multicolumn{2}{|r|}{0.81} & \\
\hline Case $(n)$ & 89 & & 61 & & 57 & & 38 & \\
\hline Model 1 & 1.00 & 0.63 & $0.44,0.90$ & 0.58 & $0.41,0.84$ & 0.37 & $0.25,0.56$ & $<0.001$ \\
\hline Model 2 & 1.00 & 0.59 & $0.41,0.85$ & 0.50 & $0.34,0.74$ & 0.28 & $0.18,0.44$ & $<0.001$ \\
\hline Total carotenoids $(\mu \mathrm{mol} / \mathrm{l})$ & 0.58 & & 0.98 & & 1.43 & & 2.34 & \\
\hline Case $(n)$ & 101 & & 59 & & 52 & & 33 & \\
\hline Model 1 & 1.00 & 0.49 & $0.34,0.69$ & 0.36 & $0.24,0.52$ & 0.26 & $0.18,0.40$ & $<0.001$ \\
\hline Model 2 & 1.00 & 0.44 & $0.31,0.64$ & 0.35 & $0.24,0.53$ & 0.19 & $0.12,0.30$ & $<0.001$ \\
\hline
\end{tabular}

* Model 1 adjusted for age, sex and energy.

† Model 2 further adjusted for marital status, education level, occupation, household income, smoking status, alcohol drinking, physical activity, serum total cholesterol, dietary intakes of fibre, cholesterol, SFA, vitamin C, vitamin E and $\beta$-carotene, and serum levels of retinol and $\alpha$-tocopherol. 


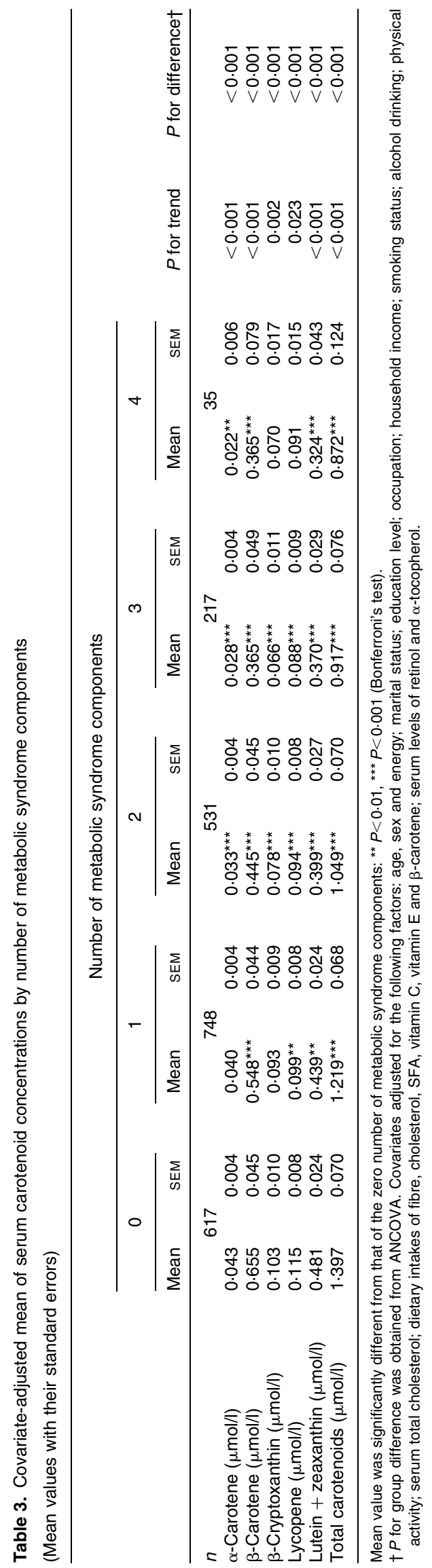

\section{Discussion}

We evaluated the relationships between serum carotenoids and the MetS in a sample of middle-aged and elderly Chinese adults and found a dose-response inverse linear association between carotenoids and the prevalence of the MetS. Furthermore, the concentration of carotenoids decreased significantly as the number of abnormal MetS components increased.

In agreement with our findings, several observational studies have found serum carotenoid concentrations to be inversely associated with the MetS ${ }^{(15,20)}$. The Third National Health and Nutrition Examination Survey (1988-94) of 8808 adults reported that those with the MetS had significantly lower concentrations of individual carotenoids (except lycopene) and total carotenoids compared with those without the MetS, after adjusting for confounding factors similar to those in the present study ${ }^{(15)}$. They also showed decreasing levels of individual carotenoids (except lycopene) and total carotenoids as the number of MetS components increased ${ }^{(15)}$. Similar results have been obtained in other cross-sectional studies $^{(20)}$. In a RCT involving 5220 adults taking a supplement containing a combination of antioxidants (vitamins $\mathrm{C}$ and $\mathrm{E}$, $\beta$-carotene, $\mathrm{Zn}$ and $\mathrm{Se}$ ), participants in the top tertile of baseline serum $\beta$-carotene levels had a $66 \%$ (range 47-79\%) lower risk of developing the MetS $(P$ for trend $<0.001)$ than those in the bottom tertile after a follow-up period of 7.5 years $^{(21)}$. However, the RCT did not observe any long-term benefits or adverse effects of $\beta$-carotene in the prevention of the $\mathrm{MetS}^{(21)}$. There are several possible explanations for the discrepancy. The population of the intervention study may have had a higher prevalence of risk factors such as smoking status and alcohol drinking, as inverse relationships have previously been found in non-smokers but not in smokers ${ }^{(19,20)}$. In the present study, the inverse associations were only observed among non-drinkers, but not among drinkers. Thus, the interaction of serum carotenoids with other risk factors should be taken into account. Furthermore, the effectiveness of individual carotenoids may rely on the levels of other carotenoids. Our findings indicated that individual carotenoids other than $\beta$-carotene were related to the MetS, suggesting that carotenoids may interact synergistically, and thus supplementing with a single $\beta$-carotene may be ineffective. Finally, $\beta$-carotene plays a role in improving antioxidant capacity only at relatively low concentrations, but loses this capacity at higher concentrations, and high-dose supplements of a single carotenoid might interfere with the absorption or bioavailability of others ${ }^{(11,32)}$.

There are several biological mechanisms accounting for the protective role of carotenoids. It is well known that increased oxidative stress plays a key role in the MetS and its components $^{(6,7)}$. Carotenoids are very efficient physical and chemical quenchers of singlet oxygen $\left({ }^{1} \mathrm{O}_{2}\right)$ and potent scavengers of other reactive oxygen species $^{(9)}$. They are also known to inhibit lipid peroxidation and to scavenge lipid peroxyl radicals, preventing the propagation of free radicalmediated chain reactions. Furthermore, carotenoids can act synergistically as an effective 'radical-trapping antioxidant' in biological membranes, thereby protecting cells against 
Table 4. Multivariate-adjusted OR of the metabolic syndrome for each quartile of serum carotenoid concentrations by subgroups of sex, smoking status and alcohol drinking*

(Odds ratios and $95 \%$ confidence intervals)

\begin{tabular}{|c|c|c|c|c|c|c|c|c|}
\hline & \multirow{2}{*}{$\frac{\text { Quartile } 1}{\text { OR }}$} & \multicolumn{2}{|c|}{ Quartile 2} & \multicolumn{2}{|c|}{ Quartile 3} & \multicolumn{2}{|c|}{ Quartile 4} & \multirow[b]{2}{*}{$P$ for interaction } \\
\hline & & OR & $95 \% \mathrm{Cl}$ & OR & $95 \% \mathrm{Cl}$ & OR & $95 \% \mathrm{Cl}$ & \\
\hline \multicolumn{9}{|l|}{$\alpha$-Carotene $(\mu \mathrm{mol} / \mathrm{l})$} \\
\hline Sex & & & & & & & & 0.203 \\
\hline Women & 1.00 & 0.76 & $0.51,1 \cdot 13$ & 0.59 & $0.39,0.90$ & 0.29 & $0.17,0.47$ & \\
\hline Men & 1.00 & 1.01 & $0.46,2 \cdot 21$ & 0.83 & $0.36,1.94$ & 0.46 & $0.17,1.27$ & \\
\hline Smoking & & & & & & & & 0.595 \\
\hline Non-smoker & 1.00 & 0.78 & $0.53,1.15$ & 0.55 & $0.37,0.83$ & 0.29 & $0.19,0.46$ & \\
\hline Smoker & 1.00 & 1.00 & $0.42,2.41$ & 0.46 & $0.13,1.65$ & 0.48 & $0 \cdot 10,2 \cdot 23$ & \\
\hline Alcohol drinking & & & & & & & & 0.048 \\
\hline No & 1.00 & 0.81 & $0.56,1.17$ & 0.54 & $0.36,0.80$ & 0.29 & $0.18,0.46$ & \\
\hline Yes & 1.00 & $2 \cdot 29$ & $0.56,9.43$ & 1.07 & $0.22,5 \cdot 20$ & 1.51 & $0.29,7.96$ & \\
\hline \multicolumn{9}{|l|}{$\beta$-Carotene $(\mu \mathrm{mol} / \mathrm{l})$} \\
\hline Sex & & & & & & & & 0.260 \\
\hline Women & 1.00 & 0.56 & $0.38,0.83$ & 0.45 & $0.29,0.69$ & 0.23 & $0.14,0.39$ & \\
\hline Men & 1.00 & 0.42 & $0.19,0.92$ & 0.21 & $0.08,0.53$ & 0.25 & $0.10,0.61$ & \\
\hline Smoking & & & & & & & & 0.507 \\
\hline Non-smoker & 1.00 & 0.60 & $0.41,0.89$ & 0.44 & $0.29,0.66$ & 0.26 & $0.16,0.41$ & \\
\hline Smoker & 1.00 & 0.42 & $0.15,1.16$ & $0 \cdot 12$ & $0.02,0.94$ & 1.05 & $0.33,3.43$ & \\
\hline Alcohol drinking & & & & & & & & 0.040 \\
\hline No & 1.00 & 0.55 & $0.38,0.80$ & 0.35 & $0.23,0.53$ & 0.21 & $0.13,0.33$ & \\
\hline Yes & 1.00 & 0.39 & $0.07,2.13$ & 0.71 & $0.13,3.85$ & 1.43 & $0.33,6 \cdot 16$ & \\
\hline \multicolumn{9}{|c|}{$\beta$-Cryptoxanthin ( $\mu \mathrm{mol} / \mathrm{l})$} \\
\hline Sex & & & & & & & & 0.328 \\
\hline Women & 1.00 & 0.91 & $0.61,1.37$ & 0.76 & $0.50,1.17$ & 0.59 & $0.37,0.94$ & \\
\hline Men & 1.00 & 0.62 & $0.27,1.42$ & 0.90 & $0.42,1.91$ & 0.33 & $0.12,0.93$ & \\
\hline Smoking & & & & & & & & 0.249 \\
\hline Non-smoker & 1.00 & 0.76 & $0.51,1.14$ & 0.72 & $0.48,1.08$ & 0.49 & $0.32,0.75$ & \\
\hline Smoker & 1.00 & 0.87 & $0.36,2.14$ & 0.53 & $0.17,1.65$ & 0.20 & $0.03,1.59$ & \\
\hline Alcohol drinking & & & & & & & & 0.693 \\
\hline No & 1.00 & 0.71 & $0.48,1.03$ & 0.66 & $0.44,0.97$ & 0.44 & $0.29,0.67$ & \\
\hline Yes & 1.00 & $2 \cdot 24$ & $0.58,8.74$ & 1.47 & $0.31,7.01$ & 0.41 & $0.04,4.14$ & \\
\hline \multicolumn{9}{|l|}{ Lycopene $(\mu \mathrm{mol} / \mathrm{l})$} \\
\hline Sex & & & & & & & & 0.116 \\
\hline Women & 1.00 & 0.73 & $0.49,1.08$ & 0.43 & $0.27,0.67$ & 0.39 & $0.25,0.61$ & \\
\hline Men & 1.00 & 0.79 & $0.35,1.81$ & 0.51 & $0.20,1.32$ & 0.85 & $0.37,1.95$ & \\
\hline Smoking & & & & & & & & 0.362 \\
\hline Non-smoker & 1.00 & 0.59 & $0.40,0.88$ & 0.45 & $0.30,0.68$ & 0.38 & $0.25,0.57$ & \\
\hline Smoker & 1.00 & 0.35 & $0 \cdot 10,1 \cdot 25$ & 0.61 & $0.20,1.90$ & 0.78 & $0.25,2.49$ & \\
\hline Alcohol drinking & & & & & & & & 0.068 \\
\hline No & 1.00 & 0.57 & $0.39,0.84$ & 0.48 & $0.32,0.71$ & 0.36 & $0.24,0.55$ & \\
\hline Yes & 1.00 & 0.91 & $0 \cdot 20,4 \cdot 18$ & $0 \cdot 30$ & $0.03,2.93$ & $2 \cdot 06$ & $0.53,7.99$ & \\
\hline \multicolumn{9}{|c|}{ Lutein+zeaxanthin $(\mu \mathrm{mol} / \mathrm{l})$} \\
\hline Sex & & & & & & & & 0.481 \\
\hline Women & 1.00 & 0.59 & $0.39,0.88$ & 0.59 & $0.39,0.90$ & 0.31 & $0.19,0.50$ & \\
\hline Men & 1.00 & $1 \cdot 19$ & $0.55,2.58$ & 0.66 & $0.27,1.59$ & 0.60 & $0.24,1.50$ & \\
\hline Smoking & & & & & & & & 0.208 \\
\hline Non-smoker & 1.00 & 0.55 & $0.37,0.81$ & 0.53 & $0.36,0.79$ & 0.30 & $0.19,0.47$ & \\
\hline Smoker & 1.00 & $1 \cdot 30$ & $0.52,3.26$ & 0.82 & $0.26,2.53$ & 0.73 & $0.22,2.47$ & \\
\hline Alcohol drinking & & & & & & & & 0.371 \\
\hline No & 1.00 & 0.57 & $0.39,0.83$ & 0.49 & $0.33,0.74$ & 0.27 & $0.17,0.43$ & \\
\hline Yes & 1.00 & 1.02 & $0.25,4.19$ & 0.72 & $0.16,3.28$ & 0.72 & $0.15,3.47$ & \\
\hline \multicolumn{9}{|c|}{ Total carotenoids $(\mu \mathrm{mol} / \mathrm{l})$} \\
\hline Sex & & & & & & & & 0.598 \\
\hline Women & 1.00 & 0.52 & $0.34,0.78$ & 0.44 & $0.29,0.69$ & 0.18 & $0.10,0.31$ & \\
\hline Men & 1.00 & 0.59 & $0.27,1.29$ & 0.30 & $0.12,0.77$ & 0.49 & $0.21,1.16$ & \\
\hline Smoking & & & & & & & & 0.852 \\
\hline Non-smoker & 1.00 & 0.50 & $0.34,0.75$ & 0.38 & $0.25,0.58$ & 0.19 & $0.12,0.31$ & \\
\hline Smoker & 1.00 & 0.21 & $0.06,0.72$ & $0 \cdot 31$ & $0.09,1.08$ & 0.71 & $0 \cdot 19,2 \cdot 61$ & \\
\hline Alcohol drinking & & & & & & & & 0.095 \\
\hline No & 1.00 & 0.50 & $0.34,0.73$ & 0.34 & $0.22,0.51$ & 0.17 & $0.11,0.28$ & \\
\hline Yes & 1.00 & 0.12 & $0.02,1.02$ & 0.74 & $0.19,2.88$ & 0.94 & $0.20,4.54$ & \\
\hline
\end{tabular}

* Model adjusted for the following factors: age, sex (except for sex stratification) and energy; marital status; education level; occupation; household income; smoking status; alcohol drinking; physical activity; serum total cholesterol, dietary intakes of fibre, cholesterol, SFA, vitamin C, vitamin E and $\beta$-carotene; serum levels of retinol and $\alpha$-tocopherol. $\alpha$ for interaction $=0.05$ /eighteen tests $=0.0028$. 
different reactive nitrogen species ${ }^{(10)}$. In addition to their antioxidant properties, carotenoids can modulate lipoxygenase enzyme activity, probably inhibiting this inflammatory enzyme and thus the production of molecules with proinflammatory properties ${ }^{(33)}$. Furthermore, carotenoids also exhibit anti-inflammatory effects by inhibiting C-reactive protein, leucocytes, fibrinogen and inflammatory cytokines ${ }^{(34,35)}$. Gouranton et al. ${ }^{(13)}$ suggested that lycopene inhibited proinflammatory cytokine production, such as IL-6, IL-1 $\beta$ and TNF- $\alpha$, to prevent obesity-associated pathologies, such as insulin resistance.

There are several limitations to the present study. First, the cross-sectional design limits the ability to draw inferences regarding temporality and causation. It is not possible to conclude from the present study whether the lower concentrations were the result of a lower consumption of dietary carotenoids or increased utilisation of carotenoids due to the oxidative stress effects of the MetS. However, a previous RCT found that the baseline concentration of $\beta$-carotene was negatively associated with the incidence of the MetS after a 7.5-year follow-up, indicating that low levels of carotenoids might be causally involved in the development of the MetS ${ }^{(21)}$. Second, participants were volunteers who might have healthier behaviours and higher incomes and education levels than the general population. However, we did not observe any significant difference in the associations studied among the subgroups stratified by smoking status, alcohol drinking, education level or income, suggesting good generalisability across different statuses of these factors. Third, we could not obtain accurate data on the participants' intake of micronutrients from supplements. However, few supplements contain these carotenoids in China. Fourth, although we adjusted for major sociodemographic characteristics, lifestyle factors, dietary intakes of antioxidants, and serum levels of $\alpha$-tocopherol and retinol simultaneously, confounding by unknown or unmeasured factors cannot be completely ruled out. Finally, the participants were middle-aged and elderly Chinese adults living in urban areas, which limits the generalisability of these results to other populations.

In general, we found an association between higher serum carotenoid levels and a lower prevalence of the MetS in Chinese adults. The present study adds to the accumulating evidence that antioxidant carotenoids may have a protective effect against the MetS. However, additional longitudinal studies and RCT are needed to confirm the beneficial association between carotenoids and the incidence of the MetS in this population.

\section{Supplementary material}

To view supplementary material for this article, please visit http://dx.doi.org/10.1017/S000711451400316X

\section{Acknowledgements}

The present study was jointly supported by the Key Program of National Natural Science Foundation of China (no. 81130052) and the 5010 Program for Clinical Researches (no. 2007032) by the Sun Yat-sen University, Guangzhou, People's Republic of China. The sponsor had no role in the design and analysis of the study or in the writing of this article.

The authors' contributions were as follows: Y.-m. C. conceived and designed the study, and critically revised the manuscript; J. L. analysed the data and wrote the paper; Y. C. participated in the laboratory assay; W-q. S., L.-p. H. and K. G. collected the data and participated in the writing of the manuscript; W.-h. L. revised the manuscript. All the authors read and approved the final version of the manuscript.

There are no conflicts of interest.

\section{References}

1. Suzuki T, Hirata K, Elkind MS, et al. (2008) Metabolic syndrome, endothelial dysfunction, and risk of cardiovascular events: the Northern Manhattan Study (NOMAS). Am Heart J 156, 405-410.

2. Reaven G (2002) Metabolic syndrome: pathophysiology and implications for management of cardiovascular disease. Circulation 106, 286-288.

3. Kereiakes DJ \& Willerson JT (2003) Metabolic syndrome epidemic. Circulation 108, 1552-1553.

4. Gu D, Reynolds K, Wu X, et al. (2005) Prevalence of the metabolic syndrome and overweight among adults in China. Lancet 365, 1398-1405.

5. He Y, Jiang B, Wang J, et al. (2006) Prevalence of the metabolic syndrome and its relation to cardiovascular disease in an elderly Chinese population. J Am Coll Cardiol 47, $1588-1594$.

6. Otani H (2011) Oxidative stress as pathogenesis of cardiovascular risk associated with metabolic syndrome. Antioxid Redox Signal 15, 1911-1926.

7. Hopps E, Noto D, Caimi G, et al. (2010) A novel component of the metabolic syndrome: the oxidative stress. Nutr Metab Cardiovasc Dis 20, 72-77.

8. Amiri F (2009) Metabolic syndrome, insulin resistance and oxidative stress: adding insights to improve cardiovascular prevention. J Hypertens 27, 1352-1354.

9. Young AJ \& Lowe GM (2001) Antioxidant and prooxidant properties of carotenoids. Arch Biochem Biophys $\mathbf{3 8 5}$ 20-27.

10. Fiedor J \& Burda K (2014) Potential role of carotenoids as antioxidants in human health and disease. Nutrients $\mathbf{6}$, 466-488.

11. Krinsky NI \& Johnson EJ (2005) Carotenoid actions and their relation to health and disease. Mol Aspects Med 26, 459-516.

12. Andersen LF, Jacobs DR Jr, Gross MD, et al. (2006) Longitudinal associations between body mass index and serum carotenoids: the CARDIA study. BrJ Nutr 95, 358-365.

13. Gouranton E, Thabuis C, Riollet C, et al. (2011) Lycopene inhibits proinflammatory cytokine and chemokine expression in adipose tissue. J Nutr Biochem 22, 642-648.

14. Marcotorchino J, Romier B, Gouranton E, et al. (2012) Lycopene attenuates LPS-induced TNF-alpha secretion in macrophages and inflammatory markers in adipocytes exposed to macrophage-conditioned media. Mol Nutr Food Res 56, 725-732.

15. Ford ES, Mokdad AH, Giles WH, et al. (2003) The metabolic syndrome and antioxidant concentrations: findings from the Third National Health and Nutrition Examination Survey. Diabetes 52, 2346-2352. 
16. Beydoun MA, Shroff MR, Chen X, et al. (2011) Serum antioxidant status is associated with metabolic syndrome among U.S. adults in recent national surveys. J Nutr 141, 903-913.

17. Beydoun MA, Canas JA, Beydoun HA, et al. (2012) Serum antioxidant concentrations and metabolic syndrome are associated among U.S. adolescents in recent national surveys. J Nutr 142, 1693-1704.

18. Sugiura M, Nakamura M, Ogawa K, et al. (2008) Associations of serum carotenoid concentrations with the metabolic syndrome: interaction with smoking. Br J Nutr 100, 1297-1306.

19. Suzuki K, Ito $\mathrm{Y}$, Inoue T, et al. (2011) Inverse association of serum carotenoids with prevalence of metabolic syndrome among Japanese. Clin Nutr 30, 369-375.

20. Coyne T, Ibiebele TI, Baade PD, et al. (2009) Metabolic syndrome and serum carotenoids: findings of a cross-sectional study in Queensland, Australia. Br J Nutr 102, 1668-1677.

21. Czernichow S, Vergnaud AC, Galan P, et al. (2009) Effects of long-term antioxidant supplementation and association of serum antioxidant concentrations with risk of metabolic syndrome in adults. Am J Clin Nutr 90, 329-335.

22. Grundy SM (2008) Metabolic syndrome pandemic. Arterioscler Thromb Vasc Biol 28, 629-636.

23. Rakugi H \& Ogihara $\mathrm{T}$ (2005) The metabolic syndrome in the Asian population. Curr Hypertens Rep 7, 103-109.

24. Povel CM, Boer JM, Reiling E, et al. (2011) Genetic variants and the metabolic syndrome: a systematic review. Obes Rev 12, 952-967.

25. Phillips C, Lopez-Miranda J, Perez-Jimenez F, et al. (2006) Genetic and nutrient determinants of the metabolic syndrome. Curr Opin Cardiol 21, 185-193.

26. Andreassi MG (2009) Metabolic syndrome, diabetes and atherosclerosis: influence of gene-environment interaction. Mutat Res 667, 35-43.
27. Ainsworth BE, Haskell WL, Herrmann SD, et al. (2011) 2011 Compendium of Physical Activities: a second update of codes and MET values. Med Sci Sports Exerc 43, 1575-1581.

28. Zhang CX \& Ho SC (2009) Validity and reproducibility of a food frequency questionnaire among Chinese women in Guangdong province. Asia Pac J Clin Nutr 18, 240-250.

29. Yang YX (2004) China Food Composition Table 2004. Beijing: Peking University Medical Press.

30. Burri BJ, Dopler-Nelson M \& Neidllinger TR (2003) Measurements of the major isoforms of vitamins $\mathrm{A}$ and $\mathrm{E}$ and carotenoids in the blood of people with spinal-cord injuries. J Chromatogr A 987, 359-366.

31. Alberti KG, Zimmet P \& Shaw J (2006) Metabolic syndrome - a new world-wide definition. A Consensus Statement from the International Diabetes Federation. Diabet Med 23, 469-480.

32. Ciccone MM, Cortese F, Gesualdo M, et al. (2013) Dietary intake of carotenoids and their antioxidant and anti-inflammatory effects in cardiovascular care. Mediators Inflamm 2013, 782137.

33. Giordano P, Scicchitano P, Locorotondo M, et al. (2012) Carotenoids and cardiovascular risk. Curr Pharm Design 18, 5577-5589.

34. Xu X-R, Zou Z-Y, Huang Y-M, et al. (2012) Serum carotenoids in relation to risk factors for development of atherosclerosis. Clin Biochem 45, 1357-1361.

35. Hozawa A, Jacobs DR Jr, Steffes MW, et al. (2007) Relationships of circulating carotenoid concentrations with several markers of inflammation, oxidative stress, and endothelial dysfunction: the Coronary Artery Risk Development in Young Adults (CARDIA)/Young Adult Longitudinal Trends in Antioxidants (YALTA) study. Clin Chem 53, 447-455. 